\title{
Laparoscopic Splenectomy for a Patient with Splenomegaly and Hypersplenism due to Idiopathic Portal Hypertension
}

Kwangho Yang, M.D. ${ }^{1}{ }^{2}$, Sung Pil Yun, M.D., Ph.D. ${ }^{3}$, Jae Hun Kim, M.D. ${ }^{3}$, Dae Hwan Kim, M.D., Ph.D. ${ }^{3}$, Hyun Sung Kim, M.D. ${ }^{1}$, Hyung II Se0, M.D., Ph.D. ${ }^{3}$

'Division of Hepato-Biliary-Pancreatic Surgery and Transplantation, Department of Surgery, Pusan National University Yangsan Hospital, ${ }^{2}$ Research Institute for Convergence of Biomedical Science and Technology, Pusan National University Yangsan Hospital, Yangsan, ${ }^{3}$ Department of Surgery, Biomedical Research Institute, Pusan National University Hospital, Busan, Korea

Idiopathic portal hypertension (IPH) is a rare disorder which is clinically characterized by portal hypertension, splenomegaly, hypersplenism and the absence of liver cirrhosis. Patients with IPH have massive splenomegaly leading to increased portal venous flow and subsequent portal hypertension. In selected IPH patients with splenomegaly and hypersplenism, splenectomy can be regarded as an effective treatment protocol for decreasing portal hypertension. We report a case of a 44-year-old woman who was diagnosed with IPH accompanied by splenomegaly and hypersplenism. She underwent laparoscopic splenectomy and clinical symptoms and hypersplenism resolved. Our study shows that laparoscopic splenectomy can be considered as a procedure for treating patients with splenomegaly and hypersplenism due to IPH.

Keywords: Idiopathic portal hypertension, Splenomegaly, Laparoscopic splenectomy
Received April 25, 2017

Revised July 19, 2017

Accepted August 1, 2017

Corresponding author

Hyung Il Seo

Department of Surgery, Biomedical Research Institute, Pusan National University Hospital, 179, Gudeok-ro, Seo-gu, Busan 49241, Korea Tel: $+82-51-240-7238$

Fax: +82-51-247-1365

E-mail: seohi71@hanmail.net

Copyright $@ 2017$ The Journal of Minimally Invasive Surgery. All rights reserved.

This is an Open Access article distributed under the terms of the Creative Commons Attribution Non-Commercial License (http:/ creativecommons.org/licenses/by-nc/4.0/) which permits unrestricted non-commercial use, distribution, and reproduction in any medium, provided the original work is properly cited.

\section{INTRODUCTION}

Idiopathic portal hypertension (IPH) is an uncommon disease presenting with clinical symptoms of portal hypertension, such as hepatomegaly, splenomegaly, hypersplenism, pancytopenia, gastric varix, and esophageal varix. ${ }^{1-4}$ Splenectomy is the recommended management procedure for these clinical symptoms of IPH. ${ }^{1,5}$ Laparoscopic splenectomy (LS) has been considered as a relative contraindication for management of massive splenomegaly, because the operation field is too narrow to perform active handling of surgical instruments. However, the improvement of surgical techniques allowed LS with minimal morbidity or mortality. Here we report a case of LS for massive splenomegaly $(26 \mathrm{~cm} / 1,517.4 \mathrm{~g})$ generated by IPH.

\section{CASE REPORT}

A 44-year old female patient visited the hospital with symptoms including chilling, fever, loss of appetite, and general weakness. In routine physical examination, there was a palpable movable mass located from the left lower abdomen to the upper abdomen. Laboratory findings revealed pancytopenia (WBC 1,030/ $\mu$, absolute neutrophil count $<500$, hemoglobin $9.2 \mathrm{~g} / \mathrm{dl}$, platelet $100,000 / \mu \mathrm{l}$ ), elevated liver enzymes (AST $123 \mathrm{IU} / \mathrm{L}$, ALT $61 \mathrm{IU} / \mathrm{L})$ and LDH (808 IU/L). Viral markers for hepatitis $\mathrm{A}, \mathrm{B}$, and $\mathrm{C}$ were all negative. Abdominal ultrasound and computed tomography (CT) revealed hepatomegaly and massive splenomegaly with multifocal splenic infarctions (Fig. 1A). Based on the results from neck CT, chest CT and bone marrow biopsy, lymphoma was ruled out from the dif- 


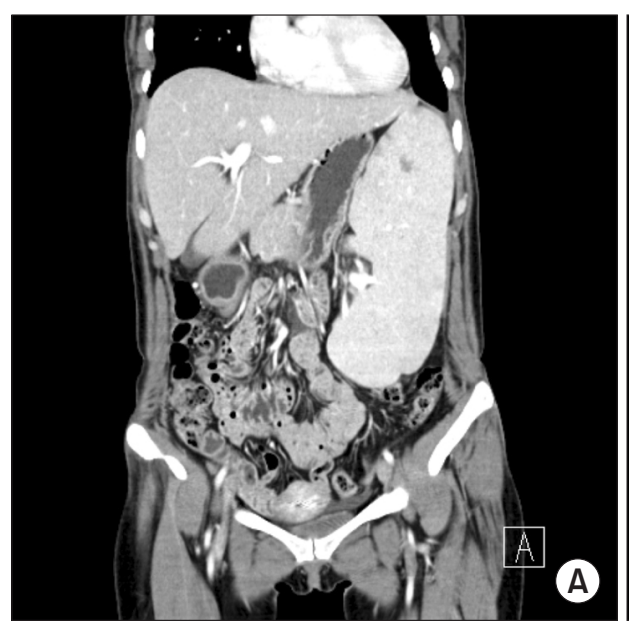

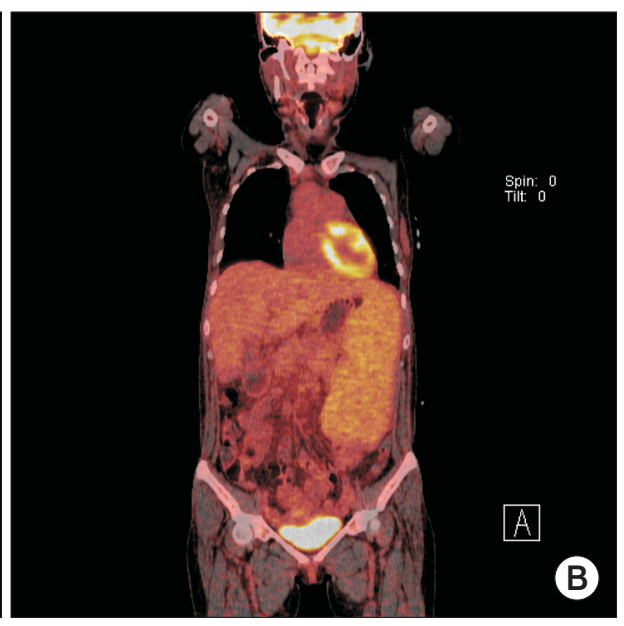

Fig. 1. (A) Initial CT finding; Contrast enhanced abdominal CT shows marked spleen enlargement with multifocal splenic infarct and suspicious of lymphoma involvement or other hematologic disorder. (B) PET/CT shows diffuse FDG uptake of spleen.

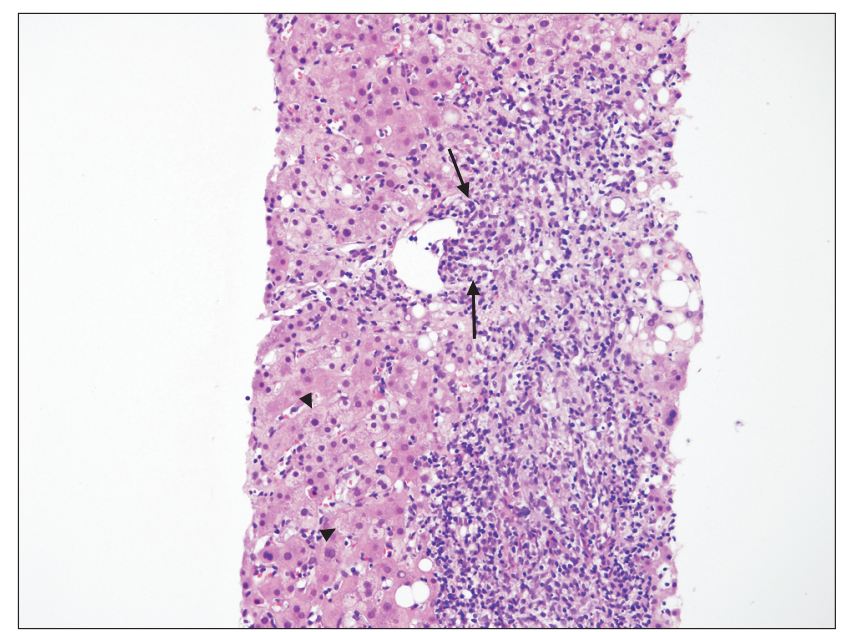

Fig. 2. The histological examination of the liver shows portal inflammation with mild periportal activity (arrow) and moderate lobular activity with ballooning degeneration (arrow head) (hematoxylin and eosin staining, $x 2001$.

ferential diagnosis. Diffuse fluorodeoxyglucose (FDG) uptake (SUVmax=3.3) was shown in positron emission tomography/ computed tomography (PET/CT) (Fig. 1B). However, esophageal or gastric varix was not found in endoscopy. Pathology report from the patient's liver biopsy confirmed chronic hepatitis with 1) portal inflammation with mild periportal activity, 2) periportal fibrosis, and 3) moderate lobular activity (Fig. 2).

Authors performed LS to treat hypersplenism due to massive splenomegaly (Fig. 3). The patient underwent operation in the supine position with 4 incisions of trocars (Fig. 4). The enlarged spleen was removed safely via $9 \mathrm{~cm}$ of left subcostal incision. The weight and the size of the isolated spleen were 1,517.4 $\mathrm{g}$ and $26.4 \times 14.8 \times 4.7 \mathrm{~cm}$, respectively (Fig. 5). There was no specific abnormal finding in pathologic evaluation. After surgery, the clinical symptoms of hypersplenism disap- peared, and pancytopenia resolved (WBC 8,160/ $\mu$, hemoglobin $13.3 \mathrm{~g} / \mathrm{dl}$, platelet $421,000 / \mu \mathrm{l})$. Six months after surgery, the patient was observed without any abnormalities.

\section{DISCUSSION}

Portal hypertension is defined as a portal-caval venous pressure gradient exceeding $5 \mathrm{mmHg}$. This clinical syndrome leads to the progression of collateral circulations and splenomegaly. ${ }^{1}$ The most important causative factor of portal hypertension is hepatic cirrhosis with underlying liver disease. However, portal hypertension can develop without hepatic cirrhosis in various conditions, and this condition is defined as idiopathic portal hypertension (IPH). IPH is generally classified into prehepatic, intrahepatic and suprahepatic portal hypertension based on the site of obstruction.,

For the diagnosis of IPH, other liver diseases that can cause portal hypertension should be ruled out. IPH is regarded as sinusoidal portal hypertension of unknown etiology. ${ }^{4}$ Liver biopsy is necessary in IPH to rule out liver diseases that cause hepatic cirrhosis. The presence of periportal fibrosis, obliterated portal venopathy, increased number of portal vascular channels and dilated portal veins herniating into the surrounding parenchyma in the absence of hepatic cirrhosis are the pathologic findings of IPH in liver biopsy., ${ }^{1,34}$ Also, as in the present case, liver biopsy can demonstrate periportal fibrosis and portal inflammation without hepatic cirrhosis.

General clinical symptoms and complications of portal hypertension can progress to IPH. Sarin et al. reported the clinical symptoms of IPH, which were splenomegaly (13.5\%), upper gastrointestinal bleeding (84.5\%), esophageal varices (92\%), and gastric varices $(22.3 \%)^{6}$ However, according to the study results in western countries and Japan, splenomegaly or abnormalities of liver enzymes are more common early symptoms of IPH than gastrointestinal bleeding. ${ }^{7,8}$ Splenomegaly in 

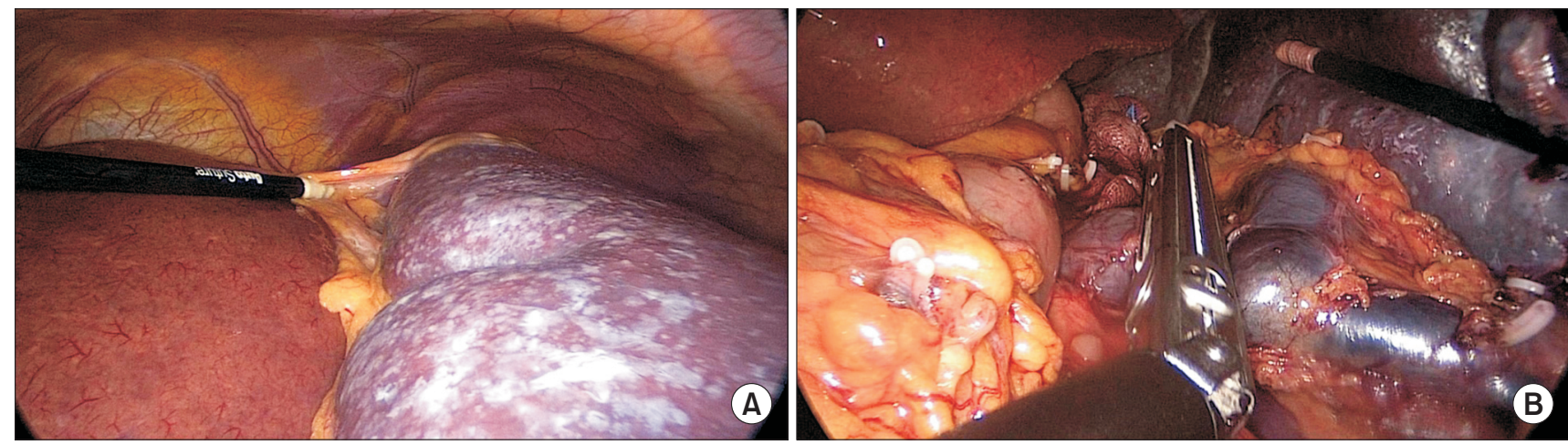

Fig. 3. (A) Gross finding seen in laparoscopic view showed massive splenomegaly. (B) Dilated splenic vein was cut with laparoscopic linear stapler.

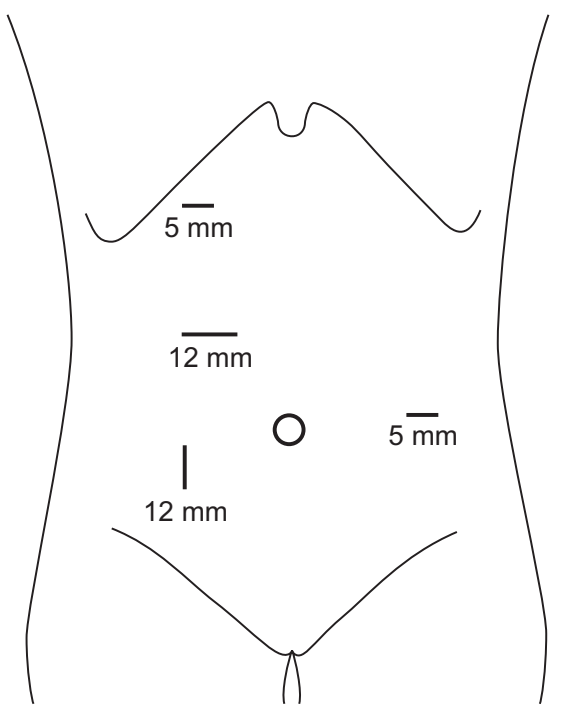

Fig. 4. The patient is positioned in supine. The surgeon work on the right side of the patient. Four trocars are used, two $5 \mathrm{~mm}$ and two $12 \mathrm{~mm}$.

IPH shows a much more severe pattern than it does in portal hypertension with hepatic cirrhosis or in portal vein thrombosis. In our case, the major clinical symptoms were splenomegaly, hypersplenism, neutropenia, anemia and no varix, which were consistent with a report conducted in Japan.

Splenectomy can relieve the clinical symptoms of hypersplenism with splenomegaly. ${ }^{1,4,9}$ Pancytopenia is the most important indicator for splenectomy in hypersplenism patients. Massive splenomegaly had been considered as a relative contraindication for LS because of technical difficulties including the narrow operation field. However, the development of laparoscopic operation devices and surgical techniques have made LS safe for massive splenomegaly. Hama et al. reported that there is no significant difference in LS and hand-assisted laparoscopic splenectomy (HALS) between patients with splenomegaly and those with a normal sized spleen. ${ }^{9}$ Wang et

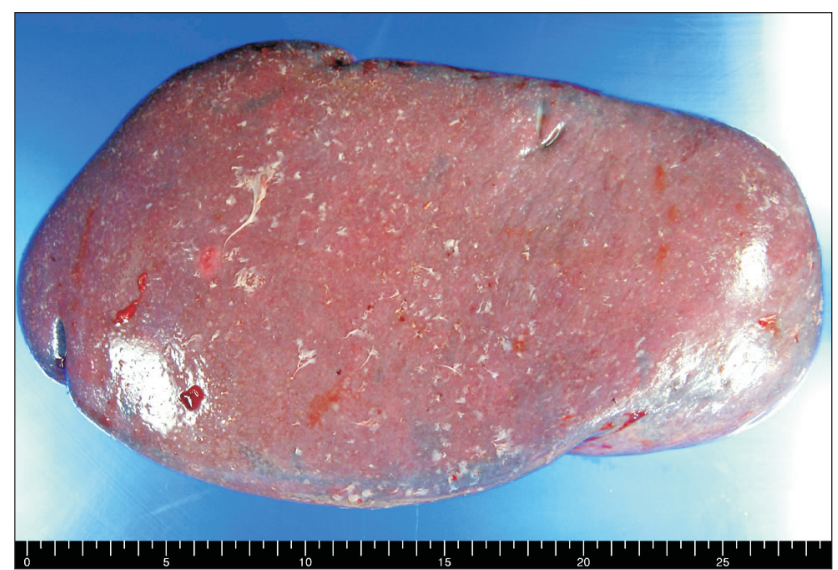

Fig. 5. Resected spleen measures 1,517.4 g in weight and $26.4 \times 14.8 \times 4.7$ $\mathrm{cm}$ in dimensions.

al. introduced a modified HALS method with an additional incision of $6 \sim 8 \mathrm{~cm}$ for removal of the enlarged spleen when the intact spleen is needed for pathologic evaluation or when conventional LS is not appropriate because of the massive size and dense adhesion around the spleen. ${ }^{10}$ In the present case, lymphoma was suspected in preoperative abdominal CT, thus, the authors performed LS with an additional $9 \mathrm{~cm}$ incision on the left subcostal region followed by intact removal of the enlarged spleen $(1,517.4 \mathrm{~g}, 26.4 \times 14.8 \times 4.7 \mathrm{~cm})$. Remission of IPH was observed after splenectomy.

Splenectomy would be an effective and curative method for IPH patients presenting with splenomegaly and hypersplenism as major symptoms. In addition, considering the advantages of laparoscopic surgery, LS should be recommended even for cases of massive splenomegaly.

\section{ACKNOWLEDGMENTS}

This work was supported by Pusan National University 
Hospital Clinical Research Fund of 2017.

\section{REFERENCES}

1) Schouten JN, Garcia-Pagan JC, Valla DC, Janssen HL. Idiopathic noncirrhotic portal hypertension. Hepatology 2011;54:1071-1081.

2) Okuda K. Non-cirrhotic portal hypertension versus idiopathic portal hypertension. J Gastroenterol Hepatol 2002;17 Suppl 3:S204-213.

3) Sarin SK, Kumar A, Chawla YK, et al. Noncirrhotic portal fibrosis/idiopathic portal hypertension: APASL recommendations for diagnosis and treatment. Hepatol Int 2007;1:398-413.

4) Harmanci $O$, Bayraktar Y. Clinical characteristics of idiopathic portal hypertension. World J Gastroenterol 2007;13:1906-1911.

5) Bolognesi M, Merkel C, Sacerdoti D, Nava V, Gatta A. Role of spleen enlargement in cirrhosis with portal hypertension. Dig Liver Dis 2002;34:144-150.
6) Sarin SK, Kapoor D. Non-cirrhotic portal fibrosis: current concepts and management. J Gastroenterol Hepatol 2002;17:526-534.

7) Hillaire $\mathrm{S}$, Bonte $\mathrm{E}$, Denninger $\mathrm{MH}$, et al. Idiopathic non-cirrhotic intrahepatic portal hypertension in the West: a re-evaluation in 28 patients. Gut 2002;51:275-280.

8) Okuda K, Kono K, Ohnishi K, et al. Clinical study of eighty-six cases of idiopathic portal hypertension and comparison with cirrhosis with splenomegaly. Gastroenterology 1984;86:600-610.

9) Hama T, Takifuji K, Uchiyama K, Tani M, Kawai M, Yamaue H. Laparoscopic splenectomy is a safe and effective procedure for patients with splenomegaly due to portal hypertension. J Hepatobiliary Pancreat Surg 2008;15:304-309.

10) Wang KX, Hu SY, Zhang GY, Chen B, Zhang HF. Hand-assisted laparoscopic splenectomy for splenomegaly: a comparative study with conventional laparoscopic splenectomy. Chin Med J (Engl) 2007;120:41-45. 